\title{
Fuzzy Foldness of P-Ideals in BCI-Algebras
}

\author{
Mahasin A. Ahmed \\ Department of Mathematics, College of Science, Sudan University of Science and Technology, Khartoum, Republic of Sudan \\ Email: mahasinaliahmed@gmail.com
}

How to cite this paper: Ahmed, M.A. (2020) Fuzzy Foldness of P-Ideals in BCI-Algebras. Journal of Applied Mathematics and Physics, 8, 2129-2141. https://doi.org/10.4236/jamp.2020.810159

Received: August 3, 2020

Accepted: October 17, 2020

Published: October 20, 2020

Copyright () 2020 by author(s) and Scientific Research Publishing Inc. This work is licensed under the Creative Commons Attribution International License (CC BY 4.0). http://creativecommons.org/licenses/by/4.0/

\section{Abstract \\ This paper aims to introduce new notions of (fuzzy) n-fold P-ideals and (fuzzy) $\mathrm{n}$-fold weak $\mathrm{P}$-ideals in $\mathrm{BCI}$-algebras, and investigate several proper- ties of the foldness theory of $\mathrm{P}$-ideals in BCI-algebras. Finally, we construct a computer-program for studying the foldness theory of P-ideals in BCI-algebras. \\ Keywords \\ BCK/BCI Algebras, P-Ideals of BCI-Algebras, Fuzzy P-Ideal of BCI-Algebra, Fuzzypoint, (Fuzzy) n-Fold P-Ideals, (Fuzzy) n-Fold Weak P-Ideals}

\section{Introduction}

The study of BCK/BCI-algebras was initiated by Iséki [1] as a generalization of the concept of set-theoretic difference and propositional calculus. Since then, a great deal of theorems has been produced on the theory of BCK/BCI-algebras. In (1965), Zadeh [2] was introduced the notion of a fuzzy subset of a set as a method for representing uncertainty. In 1991, Xi [3] defined fuzzy subsets in BCK/BCI-algebras.

Huang and Chen [4] introduced the notions of $\mathrm{n}$-fold implicative ideal and n-fold (weak) commutative ideals. Y. B. Jun [5] discussed the fuzzification of n-fold positive implicative, commutative, and implicative ideal of BCK-algebras.

In this paper, we redefined a P-ideal of BCI-algebras and studied the foldness theory of fuzzy P-ideals, P-weak ideals, fuzzy weak P-ideals, and weak P-weak ideals in BCI-algebras. This theory can be considered as a natural generalization of P-ideals. Indeed, given any BCI-algebras $X$, we use the concept of fuzzy point to characterize $\mathrm{n}$-fold P-ideals in $X$. Finally, we construct some algorithms for studying the foldness theory of P-ideals in BCI-algebras. 


\section{Preliminaries}

Here we include some elementary aspects of BCI that are necessary for this paper. For more detail, we refer to [4] [6].

An algebra $(X ; *, 0)$ of type $(2,0)$ is called BCI-algebra if

$\forall x, y, z \in X$ the following conditions hold:

BCI-1. $((x * y) *(x * z)) *(z * y)=0$;

BCI-2. $(x *(x * y)) * y=0$;

BCI-3. $x * x=0$;

BCI-4. $x * y=0$ and $y * x=0 \Rightarrow x=y$.

A binary relation $\leq$ can be defined by

BCI-5. $x \leq y \Leftrightarrow x * y=0$.

Then $(X, \leq)$ is a partially ordered set with least element 0 .

The following properties also hold in any BCI-algebra [7] [8]:

1) $x * 0=x$

2) $x * y=0$ and $y * z=0 \Rightarrow x * z=0$;

3) $x * y=0 \Rightarrow(x * z) *(y * z)=0$ and $(z * y) *(z * x)=0$;

4) $(x * y) * z=(x * z) * y$;

5) $(x * y) * x=0$;

6) $x *(x *(x * y))=x * y$; let $(X, *, 0)$ be a BCI-algebra.

Definition 2.1. A fuzzy subset of a BCK/BCI-algebra $X$ is a function $\mu: X \rightarrow[0,1]$.

Definition 2.2. (C. Lele, C. Wu, P. Weke, T. Mamadou, and C.E. Njock [9]). Let $\xi$ be the family of all fuzzy sets in $X$. For $x \in X$ and $\lambda \in(0,1], \quad x_{\lambda} \in \xi$ is a fuzzy point if

$$
x_{\lambda}(y)= \begin{cases}\lambda & \text { if } x=y \\ 0 & \text { otherwise }\end{cases}
$$

We denote by $\tilde{X}=\left\{x_{\lambda}: x \in X, \lambda \in(0,1]\right\}$ the set of all fuzzy points on $X$, and we define a binary operation on $\tilde{X}$ as follows

$$
x_{\lambda} * y_{\mu}=(x * y)_{\min (\lambda, \mu)}
$$

It is easy to verify $\forall x_{\lambda}, y_{\mu}, z_{\alpha} \in \tilde{X}$, the following conditions hold:

BCI-1'. $\left(\left(x_{\lambda} * y_{\mu}\right) *\left(x_{\lambda} * z_{\alpha}\right)\right) *\left(z_{\alpha} * y_{\mu}\right)=0_{\min (\lambda, \mu, \alpha)}$;

BCI-2'. $\left(x_{\lambda} *\left(x_{\lambda} * y_{\mu}\right)\right) * y_{\mu}=0_{\min (\lambda, \mu)}$;

BCI-3'. $x_{\lambda} * x_{\mu}=0_{\min (\lambda, \mu)}$;

BCK-5'. $0_{\mu} * x_{\lambda}=0_{\min (\lambda, \mu)}$.

Remark 2.3. (C. Lele, C. Wu, P. Weke, T. Mamadou, and C.E. Njock [9]). The condition BCI-4 is not true $(\tilde{X}, *)$. So the partial order $\leq(X, *)$ cannot be extended to $(\tilde{X}, *)$.

We can also establish the following conditions $\forall x_{\lambda}, y_{\mu}, z_{\alpha} \in \tilde{X}$ :

1') $x_{\lambda} * 0_{\mu}=x_{\min (\lambda, \mu)}$

2) $x_{\lambda} * y_{\mu}=0_{\min (\lambda, \mu)}$ and $y_{\mu} * z_{\alpha}=0_{\min (\mu, \alpha)} \Rightarrow x_{\lambda} * z_{\alpha}=0_{\min (\lambda, \alpha)}$; 
3') $\left.x_{\lambda} * y_{\mu}=0_{\min (\lambda, \mu)} \Rightarrow\left(x_{\lambda} * z_{\alpha}\right)\right) *\left(y_{\mu} * z_{\alpha}\right)=0_{\min (\lambda, \mu, \alpha)}$ and

$\left(z_{\alpha} * y_{\mu}\right) *\left(z_{\alpha} * x_{\lambda}\right)=0_{\min (\lambda, \mu, \alpha)} ;$

4') $\left(x_{\lambda} * y_{\mu}\right) * z_{\alpha}=\left(x_{\lambda} * z_{\alpha}\right) * y_{\mu}$;

5) $\left(x_{\lambda} * y_{\mu}\right) * x_{\lambda}=0_{(\lambda, \mu)}$;

6) $x_{\lambda} *\left(x_{\lambda} *\left(x_{\lambda} * y_{\mu}\right)\right)=x_{\lambda} * y_{\mu}$.

We recall that if $A$ is a fuzzy subset of a BCK/BCI algebra $X$, then we have the following:

$$
\begin{gathered}
\tilde{A}=\left\{x_{\lambda} \in \tilde{X}: A(x) \geq \lambda, \lambda \in(0,1]\right\} . \\
\forall \lambda \in(0,1], \tilde{X}_{\lambda}=\left\{x_{\lambda}: x \in X\right\} \text {, and } \tilde{A}_{\lambda}=\left\{x_{\lambda} \in \tilde{X}_{\lambda}: A(x) \geq \lambda\right\}
\end{gathered}
$$

We also have $\tilde{X}_{\lambda} \subseteq \tilde{X}, \tilde{A} \subseteq \tilde{X}, \tilde{A}_{\lambda} \subseteq \tilde{A}, \tilde{A}_{\lambda} \subseteq \tilde{X}_{\lambda}$, and one can easily check that $\left(\tilde{X}_{\lambda} ; *, 0_{\lambda}\right)$ it is a BCK-algebra.

Definition 2.4 (Isèki [10]). A nonempty subset of BCK/BCI-algebra $X$ is called an ideal of $X$ if it satisfies
1) $0 \in I$
2) $\forall x, y \in X,(x * y \in I$ and $y \in I) \Rightarrow x \in I$.

Definition 2.5. A nonempty subset $I$ of BCI-algebra $X$ is P-ideal if it satisfies:

1) $0 \in I$;

2) $\forall x, y, z \in X$

$$
((x * z) *(y * z) \in I \text { and } y \in I) \Rightarrow x \in I
$$

Definition 2.6 (Xi [11]). A fuzzy subset $A$ of a BCK/BCI algebra $X$ is a fuzzy ideal if

1) $\forall x \in X, A(0) \geq A(x)$;

2) $\forall x, y \in X, A(x) \geq \min (A(x * y), A(y))$.

Definition 2.7 (Xi [11]). A fuzzy subset $A$ of a BCI-algebra $X$ is called a fuzzy P-ideal of $X$ if.

1) $\forall x \in X, A(0) \geq A(x)$;

2) $\forall x, y, z \in X$

$$
A(x) \geq \min (A((x * z) *(y * z)) * A(y))
$$

Definition $2.8[12] . \quad \tilde{A}$ is a weak ideal of $\tilde{X}$ if

1) $\forall v \in \operatorname{Im}(A) ; 0_{v} \in \tilde{A}$;

2) $\forall x_{\lambda}, y_{\mu} \in X$. Such that $x_{\lambda} * y_{\mu} \in \tilde{A}$ and $y_{\mu} \in \tilde{A}$, we have

$$
x_{\min (\lambda, \mu)} \in \tilde{A} \text {. }
$$

Theorem 2.9 [13]. Suppose that $A$ is a fuzzy subset of a BCK-algebra $X$, then the following conditions are equivalent:

1) $A$ is a fuzzy ideal;

2) $\forall x_{\lambda}, y_{\mu} \in \tilde{A},\left(z_{\alpha} * y_{\mu}\right) * x_{\lambda}=0_{\min (\lambda, \mu, \alpha)} \Rightarrow z_{\min (\lambda, \mu, \alpha)} \in \tilde{A}$; 
3) $\forall t \in(0,1]$, the $t$-level subset $A^{t}=\{x \in X: A(x) \geq t\}$ in an ideal when $A^{t} \neq \phi$;

4) $\tilde{A}$ is a weak ideal.

\section{Fuzzy n-Fold P-Ideals in BCI-Algebras}

Throughout this paper $\tilde{X}$ is the set of fuzzy points on BCI-algebra $X$ and $n \in \mathbb{N}$ (where $\mathbb{N}$ the set of all the natural numbers).

Let us denote $(\cdots((x * y) * y) * \cdots) * y$ by $x * y^{n}$.

Moreover, $\left(\cdots\left(\left(x_{\min (\lambda, \mu)} * 0_{\mu}\right) * 0_{\mu}\right) * \cdots\right) * 0_{\mu}$ by $x_{\lambda} * y_{\mu}^{n}$ (where $y$ and $y_{\mu}$ occurs respectively $\mathrm{n}$ times) with $x, y \in X, x_{\lambda}, y_{\lambda} \in \tilde{X}$.

Definition 3.1. A nonempty subset $I$ of a BCI-algebra $X$ is an n-fold P-ideal of $X$ if it satisfies :

1) $0 \in I$

2) $\forall x, y, z \in X$

$$
((x * z) *(y * z) \in I \text { and } y \in I) \Rightarrow x * z^{n} \in I .
$$

Definition 3.2. A fuzzy subset $A$ of $X$ is called a fuzzy n-fold P-ideal of $X$ if it satisfies :

1) $\forall x \in X, A(0) \geq A(x)$;

2) $\forall x, y, z \in X$,

$$
A\left(x * z^{n}\right) \geq \min (A((x * z) *(y * z)) * A(y)) .
$$

Definition 3.3. $\tilde{A}$ is P-weak ideal of $\tilde{X}$ if

1) $\forall v \in \operatorname{Im}(A), 0_{v} \in \tilde{A}$;

2) $\forall x_{\lambda}, y_{\mu}, z_{\alpha} \in \tilde{X}$,

$$
\left(\left(x_{\lambda} * z_{\alpha}\right) *\left(y_{\mu} * z_{\alpha}\right) \in \tilde{A} \text { and } y_{\mu} \in \tilde{A}\right) \Rightarrow x_{\min (\lambda, \mu)} * z_{\alpha} \tilde{A} .
$$

Definition 3.4. $\tilde{A}$ is an $\mathrm{n}$-fold $\mathrm{P}$-weak ideal of $\tilde{X}$ if

1) $\forall v \in \operatorname{Im}(A), 0_{v} \in \tilde{A}$;

2) $\forall x_{\lambda}, y_{\mu}, z_{\alpha} \in \tilde{X}$,

$$
\left(\left(x_{\lambda} * z_{\alpha}\right) *\left(y_{\mu} * z_{\alpha}\right) \in \tilde{A} \text { and } y_{\mu} \in \tilde{A}\right) \Rightarrow x_{\min (\lambda, \mu)} * z_{\alpha}^{n} \in \tilde{A} .
$$

Example 3.5. Let $X=\{0, a, b, c\}$ with * defined by Table 1 .

By simple computations, one can prove that $(X, *, 0)$ is BCI-algebra. Define $\mu: X \rightarrow[0,1]$ by $\mu(0)=1, \mu(a)=\mu(b)=\mu(c)=t$, where $t \in[0,1]$.

Table 1. Example 3.5.

\begin{tabular}{ccccc}
\hline$*$ & 0 & $a$ & $b$ & $c$ \\
\hline 0 & 0 & 0 & 0 & $c$ \\
\hline$a$ & $a$ & 0 & 0 & $c$ \\
$c$ & $b$ & $b$ & 0 & $c$ \\
\\
$c$ & $c$ & $c$ & $c$ & 0 \\
\hline
\end{tabular}


One can easily check that for any $n \geq 3$.

Is a fuzzy $\mathrm{n}$-fold P-ideal.

Remark 3.6. $\tilde{A}$ is a 1-fold P-weak ideal of a BCK-algebra $\tilde{X}$ if $\tilde{A}$ is P-weak ideal of $\tilde{X}$.

Theorem 3.7. If $A$ is a fuzzy subset of $X$, then $A$ is a fuzzy n-fold P-ideal if $\tilde{A}$ is an $\mathrm{n}$-fold P-weak ideal.

Proof. $\Rightarrow$

- Let $\lambda \in \operatorname{Im}(A)$, it is easy to prove that $0_{\lambda} \in \tilde{A}$;

- Let $\left(x_{\lambda} * z_{\alpha}\right) *\left(y_{\mu} * z_{\alpha}\right) \in \tilde{A}$ and $y_{\mu} \in \tilde{A}$ $A((x * z) *(y * z)) \geq \min (\lambda, \mu, \alpha)$ and $A(y) \geq \mu$.

Since $A$ is a fuzzy n-fold P-ideal, we have

$$
\begin{aligned}
A\left(x * z^{n}\right) & \geq \min (A((x * z) *(y * z)) * A(y)) \\
& \geq \min (\min (\lambda, \mu, \alpha), \mu)=\min (\lambda, \mu, \alpha)
\end{aligned}
$$

Therefore $\left(x * z^{n}\right)_{\min (\lambda, \mu, \alpha)}=x_{\min (\lambda, \mu)} * z_{\alpha}^{n} \in \tilde{A}$.

$\Leftarrow$

- Let $x \in X$, it is easy to prove that $A(0) \geq A(x)$;

- Let $x, y, z \in X$ and let $A((x * z) *(y * z))=\beta$ and $A(y)=\alpha$, then $((x * z) *(y * z))_{\min (\beta, \alpha)}=\left(\left(x_{\beta} * z_{\alpha}\right) *\left(y_{\alpha} * z_{\alpha}\right)\right) \in \tilde{A}$ and $y_{\alpha} \in \tilde{A}$.

Since $\tilde{A}$ is P-weak ideal, we have

$$
x_{\min (\beta, \alpha)} * z_{\alpha}^{n}=\left(x * z^{n}\right)_{\min (\beta, \alpha)} \in \tilde{A}
$$

Thus $A\left(x * z^{n}\right) \geq \min (\beta, \alpha)=\min (A((x * z) *(y * z)), A(y))$.

Proposition 3.8. An $\mathrm{n}$-fold $\mathrm{P}$-weak ideal is a weak ideal.

Proof. $\forall x_{\lambda}, y_{\mu} \in \tilde{X}$ let $x_{\lambda} * y_{\mu}=\left(x_{\lambda} * 0_{\mu}\right) *\left(y_{\mu} * 0_{\mu}\right) \in \tilde{A}$ and $y_{\mu} \in \tilde{A}$, since $\tilde{A} \mathrm{n}$-fold P-weak ideal, we have

$$
x_{\min (\lambda, \mu)}=x_{\min (\lambda, \mu)} * 0_{\mu}^{n} \in \tilde{A}
$$

Thus $\tilde{A}$ is a weak ideal.

Corollary 3.9. A fuzzy $n$-fold P-ideal is a fuzzy ideal.

Theorem 3.10. Let $\left\{\tilde{A}_{i \in I}\right\}$ be a family of $n$-fold P-weak ideals and $\left\{A_{i \in I}\right\}$ be a family of fuzzy-fold P-ideals. Then: 1) $\bigcap_{i \in I} \tilde{A}_{i}$ is an n-fold P-weak ideal.

2) $\bigcup_{i \in I} \tilde{A}_{i}$ is an n-fold P-weak ideal.

3) $\bigcap_{i \in I} A_{i}$ is a fuzzy $n$-fold $P$-ideal.

4) $\bigcup_{i \in I} A_{i}$ is a fuzzy $n$-fold P-ideal.

Proof. 1) $\forall \lambda \in \operatorname{Im}\left(\bigcap_{i \in I} \tilde{A}_{i}\right)$, then $\lambda \in \operatorname{Im}\left(\tilde{A}_{i}\right), \forall i$, so, $0_{\lambda} \in \tilde{A}_{i}, \forall i$, i.e. $0_{\lambda} \in \bigcap_{i \in I} \tilde{A}_{i}$. For every $x_{\mu}, y_{\lambda}, z_{\alpha} \in \tilde{X}$, if $\left(x_{\lambda} * z_{\alpha}\right) *\left(y_{\mu} * z_{\alpha}\right) \in \bigcap_{i \in I} \tilde{A}_{i}$ and $y_{\mu} \in \bigcap_{i \in I} \tilde{A}_{i}$, then

$$
\left(x_{\lambda} * z_{\alpha}\right) *\left(y_{\mu} * z_{\alpha}\right) \in \tilde{A}_{i} \text { and } y_{\mu} \in \tilde{A}_{i} \quad \forall i \text {, thus }
$$




$$
x_{\min (\lambda, \mu)} * z_{\alpha}^{n} \in \tilde{A}_{i}, \forall i
$$

So $x_{\min (\lambda, \mu)} * z_{\alpha}^{n} \in \bigcap_{i \in I} \tilde{A}_{i}$. Thus $\bigcap_{i \in I} \tilde{A}_{i}$ is an n-fold P-weak ideals.

2) $\forall \lambda \in \operatorname{Im}\left(\bigcup_{i \in I} \tilde{A}_{i}\right)$, then $\exists i_{0} \in I$, such that $\lambda \in \tilde{A}_{i_{0}}$, so, $0_{\lambda} \in \tilde{A}_{i_{0}}$, i.e. $0_{\lambda} \in \bigcup_{i \in I} \tilde{A}_{i}$. For every $x_{\mu}, y_{\lambda}, z_{\alpha} \in \tilde{X}$, if $\left(x_{\lambda} * z_{\alpha}\right) *\left(y_{\mu} * z_{\alpha}\right) \in \bigcup_{i \in I} \tilde{A}_{i}$ and $y_{\mu} \in \bigcup_{i \in I} \tilde{A}_{i}$, then $\exists i_{0} \in I$ such that

$$
\left(x_{\lambda} * z_{\alpha}\right) *\left(y_{\mu} * z_{\alpha}\right) \in \tilde{A}_{i_{0}} \text { and } y_{\mu} \in \tilde{A}_{i_{0}} \text {, thus } x_{\min (\lambda, \mu)} * z_{\alpha}^{n} \in \tilde{A}_{i_{0}} .
$$

So $x_{\min (\lambda, \mu)} * z_{\alpha}^{n} \in \bigcup_{i \in I} \tilde{A}_{i}$. Thus $\bigcup_{i \in I} \tilde{A}_{i}$ is an n-fold P-weak ideals.

3) Follows from 1) and Theorem 3.7.

4) Follows from 2) and Theorem 3.7.

\section{Fuzzy-Fold Weak P-Ideals in BCI-Algebras}

In this section, we define and give some characterizations of (fuzzy) n-fold weak P-weak ideals in BCI-algebras.

Definition 4.1. A nonempty subset $I$ of $X$ is called an n-fold weak P-ideal of $X$ if it satisfies

1) $0 \in I$;

2) $\forall x, y, z \in X\left((x * z) *\left(y * z^{n}\right) \in I\right.$ and $\left.y \in I\right) \Rightarrow x \in I$.

Definition 4.2. A fuzzy subset $A$ of $X$ is called a fuzzy n-fold weak P-ideal of $X$ if it satisfies

1) $\forall x \in X, A(0) \geq A(x)$;

2) $\forall x, y, z, A(x) \geq \min \left(A\left((x * z) *\left(y * z^{n}\right)\right), A(y)\right)$.

Definition 4.3. $\tilde{A}$ is a weak P-weak ideal of $\tilde{X}$ if

1) $\forall v \in \operatorname{Im}(A), 0_{v} \in \tilde{A}$;

2) $\forall x_{\lambda}, y_{\mu}, z_{\alpha} \in \tilde{X}$

$$
\left(\left(x_{\lambda} * z_{\alpha}\right) *\left(y_{\mu} * z_{\alpha}\right) \in \tilde{A} \text { and } y_{\mu} \in \tilde{A}\right) \Rightarrow x_{\min (\lambda, \mu, \alpha)} \in \tilde{A} .
$$

Definition 4.4. $\tilde{A}$ is an $\mathrm{n}$-fold a weak $\mathrm{P}$-weak ideal of $\tilde{X}$ if

1) $\forall v \in \operatorname{Im}(A), 0_{v} \in \tilde{A}$;

2) $\forall x_{\lambda}, y_{\mu}, z_{\alpha} \in \tilde{X}$,

$$
\left(\left(x_{\lambda} * z_{\alpha}\right) *\left(y_{\mu} * z_{\alpha}^{n}\right) \in \tilde{A} \text { and } y_{\mu} \in \tilde{A}\right) \Rightarrow x_{\min (\lambda, \mu, \alpha)} \in \tilde{A} .
$$

Example 4.5. Let $X=\{0,1,2,3\}$ in which $*$ is given by Table 2 .

Table 2. Example 4.5.

\begin{tabular}{ccccc}
\hline$*$ & 0 & $a$ & $b$ & $c$ \\
\hline 0 & 0 & 0 & 0 & 0 \\
$a$ & $a$ & 0 & 0 & 0 \\
$b$ & $b$ & $b$ & 0 & 0 \\
$c$ & $c$ & $c$ & $c$ & 0 \\
\hline
\end{tabular}


Then $(X ; *, 0)$ is a BCI-algebra. Let $t_{1}, t_{2} \in(0,1]$ and let us define a fuzzy subset $A: X \rightarrow[0,1]$ by

$$
t_{1}=A(0)=A(a)=A(b)>A(c)=t_{2}
$$

It is easy to check that for any $n>2$

$$
\tilde{A}=\left\{0_{\lambda}: \lambda \in\left(0, t_{1}\right]\right\} \cup\left\{a_{\lambda}: \lambda \in\left(0, t_{2}\right]\right\} \cup\left\{b_{\lambda}: \lambda \in\left(0, t_{1}\right]\right\} \cup\left\{c_{\lambda}: \lambda \in\left(0, t_{2}\right]\right\}
$$

Is an $\mathrm{n}$-fold weak $\mathrm{P}$-weak ideal.

Remark 4.6. $\tilde{A}$ is a 1-fold weak P-weak ideal of a BCK-algebra $X$ if $\tilde{A}$ is a weak P-weak ideal.

Theorem 4.7. [13] If $A$ is a fuzzy subset of $X$, then $A$ is a fuzzy n-fold weak P-ideal if $\tilde{A}$ is an $\mathrm{n}$-fold weak P-weak ideal.

Proof. $\Rightarrow$

- Let $\lambda \in \operatorname{Im}(A)$ obviously $0_{\lambda} \in \tilde{A}$;

- Let $\left(x_{\lambda} * z_{\alpha}\right) *\left(y_{\mu} * z_{\alpha}^{n}\right) \in \tilde{A}$ and $y_{\mu} \in \tilde{A}$, then

$A\left(\left(x_{\lambda} * z_{\alpha}\right) *\left(y_{\mu} * z_{\alpha}^{n}\right)\right) \geq \min (\lambda, \mu, \alpha)$ and $A(y) \geq \mu$.

Since $A$ is a fuzzy n-fold weak P-ideal, we have

$$
\begin{aligned}
\forall x, y, z, A(x) & \geq \min \left(A\left((x * z) *\left(y * z^{n}\right)\right), A(y)\right) \\
& \geq \min (\min (\lambda, \mu, \alpha), \alpha)=\min (\lambda, \mu, \alpha)
\end{aligned}
$$

Therefore $x_{\min (\lambda, \mu, \alpha)} \in \tilde{A}$.

$\Leftarrow$

- Let $x \in X$, it is easy to prove that $A(0) \geq A(x)$;

- Let $\forall x, y, z, A\left((x * z) *\left(y * z^{n}\right)\right)=\beta$ and $A(y)=\alpha$.

Then $\left((x * z) *\left(y * z^{n}\right)\right)_{\min (\beta, \alpha)}=\left(\left(x_{\beta} * z_{\beta}\right) *\left(y_{\alpha} * z_{\beta}^{n}\right)\right) \in \tilde{A}$ and $y_{\alpha} \in \tilde{A}$.

Since $\tilde{A}$ is $\mathrm{n}$-fold weak P-weak ideal, we have

$$
x_{\min (\beta, \alpha)} \in \tilde{A}
$$

Hence $A(x) \geq \min (\beta, \alpha)=\min \left(A\left((x * z) *\left(y * z^{n}\right)\right), A(y)\right)$.

Proposition 4.8. An $\mathrm{n}$-fold weak $\mathrm{P}$-weak ideal is a weak ideal.

Proof. Let $x_{\lambda}, y_{\mu} \in \tilde{X}$ and $x_{\lambda} * y_{\mu}=\left(x_{\lambda} * 0_{\mu}\right) *\left(y_{\mu} * 0_{\mu}^{n}\right) \in \tilde{A}$ and $y_{\mu}$.

Since $\tilde{A}$ is $\mathrm{n}$-fold weak P-weak ideal, we have $x_{\min (\lambda, \mu)} \in \tilde{A}$.

Corollary 4.9. A fuzzy $n$-fold weak P-ideal is a fuzzy ideal.

Theorem 4.10. Let $\left\{\tilde{A}_{i \in I}\right\}$ be a family of $\mathrm{n}$-fold weak P-weak ideals and $\left\{A_{i \in I}\right\}$ be a family of fuzzy $\mathrm{n}$-fold weak P-ideals. then 1) $\bigcap_{i \in I} \tilde{A}_{i}$ is an $\mathrm{n}$-fold weak P-weak ideal.

2) $\bigcup_{i \in I} \tilde{A}_{i}$ is an $\mathrm{n}$-fold weak P-weak ideal.

3) $\bigcap_{i \in I} A_{i}$ is a fuzzy $n$-fold weak $\mathrm{P}$-ideal.

4) $\bigcup_{i \in I} A_{i}$ is a fuzzy n-fold weak P-ideal. 
Proof. 1) $\forall \lambda \in \operatorname{Im}\left(\bigcap_{i \in I} \tilde{A}_{i}\right)$, then $\lambda \in \operatorname{Im}\left(\tilde{A}_{i}\right), \forall i$, so, $0_{\lambda} \in \tilde{A}_{i}, \forall i$, i.e. $0_{\lambda} \in \bigcap_{i \in I} \tilde{A}_{i}$. For every $x_{\mu}, y_{\lambda}, z_{\alpha} \in \tilde{X}$, if

$$
\begin{gathered}
\left(x_{\lambda} * z_{\alpha}\right) *\left(y_{\mu} * z_{\alpha}^{n}\right) \in \bigcap_{i \in I} \tilde{A}_{i} \text { and } y_{\mu} \in \bigcap_{i \in I} \tilde{A}_{i}, \text { then } \\
\left(x_{\lambda} * z_{\alpha}\right) *\left(y_{\mu} * z_{\alpha}^{n}\right) \in \tilde{A}_{i} \text { and } y_{\mu} \in \tilde{A}_{i} \quad \forall i, \text { thus } \\
x_{\min (\lambda, \mu, \alpha)} \in \tilde{A}_{i}, \forall i
\end{gathered}
$$

So $x_{\min (\lambda, \mu, \alpha)} \in \bigcap_{i \in I} \tilde{A}_{i}$. Thus $\bigcap_{i \in I} \tilde{A}_{i}$ is an n-fold weak P-weak ideal.

2) $\forall \lambda \in \operatorname{Im}\left(\bigcup_{i \in I} \tilde{A}_{i}\right)$, then $\exists i_{0} \in I$, such that $\lambda \in \tilde{A}_{i_{0}}$, so, $0_{\lambda} \in \tilde{A}_{i_{0}}$, i.e. $0_{\lambda} \in \bigcup_{i \in I} \tilde{A}_{i}$. For every $x_{\mu}, y_{\lambda}, z_{\alpha} \in \tilde{X}$, if $\left(x_{\lambda} * z_{\alpha}\right) *\left(y_{\mu} * z_{\alpha}^{n}\right) \in \bigcup_{i \in I} \tilde{A}_{i}$ and $y_{\mu} \in \bigcup_{i \in I} \tilde{A}_{i}$, then $\exists i_{0} \in I$ such that

$$
\left(x_{\lambda} * z_{\alpha}\right) *\left(y_{\mu} * z_{\alpha}^{n}\right) \in \tilde{A}_{i_{0}} \text { and } y_{\mu} \in \tilde{A}_{i_{0}} \text {, thus } x_{\min (\lambda, \mu, \alpha)} \in \tilde{A}_{i_{0}} \text {. }
$$

So $x_{\min (\lambda, \mu, \alpha)} \in \bigcup_{i \in I} \tilde{A}_{i}$. Thus $\bigcup_{i \in I} \tilde{A}_{i}$ is an n-fold weak P-weak ideal.

3) Follows from 1) and Theorem 4.7.

4) Follows from 2) and Theorem4.7.

\section{Algorithms}

Here we give some algorithms for studding the structure of the foldness of (fuzzy) P-ideals In BCI-algebras

\section{Algorithm for AP-Ideals of BCI-Algebra}

Input $(X: B C I$-algebra, *: binary operation, $I$ : the subset of $X)$;

Output(“ $I$ is aP-ideal of $X$ or not");

Begin

If $I=\phi$ then

go to (1.);

EndIf

If $0 \notin I$ then

go to (1.);

EndIf

Stop:=false;

$i:=1$;

While $i \leq|X|$ and not (Stop) do $j:=1$;

While $j \leq|X|$ and not (Stop) do $k:=1$;

While $k \leq|X|$ and not (Stop) do

If $\left(x_{i} * z_{k}\right) *\left(y_{j} * z_{k}\right) \in I$ and $y_{j} \in I$ then If $x_{i} \notin I$

Stop:=true;

EndIf 


\section{EndIf}

Endwhile

Endwhile

Endwhile

If Stop then

Output (“ $I$ is aP-ideal of $X$ ”)

Else

(1.) Output (" $I$ is not aP-ideal of $X "$ )

EndIf

End

\section{Algorithm for $\mathbf{n}$-fold P-Ideals of BCI-Algebra}

Input $(X: B C I$-algebra, * : binary operation, $I$ : a subset of $X)$;

Output(" $I$ is n-fold P-ideal of $X$ or not");

Begin

If $I=\phi$ then go to (1.);

EndIf

If $0 \notin I$ then go to (1.);

EndIf

Stop:=false,

$i:=1$;

While $i \leq|X|$ and not (Stop) do $j:=1$;

While $j \leq|X|$ and not (Stop) do $k:=1$;

While $k \leq|X|$ and not (Stop) do

If $\left(x_{i} * z_{k}\right) *\left(y_{j} * z_{k}\right) \in I$ and $y_{j} \in I$ then If $x_{i} * z_{k}^{n} \notin I$

Stop:=true;

EndIf

EndIf

Endwhile

Endwhile

Endwhile

If Stop then

Output (" $I$ is ann-fold P-ideal of $X$ ")

Else

(1.) Output (" $I$ is not ann-fold P-ideal of $X$ ")

EndIf

End

Algorithm for Fuzzy P-Ideals of BCI-Algebra

Input $(X: B C I$-algebra, *: binary operation, $A$ : the fuzzy subset of $X)$; 
Output(" $A$ is a fuzzy P-ideal of $X$ or not");

Begin

Stop:=false,

$i:=1$;

While $i \leq|X|$ and not (Stop) do

If $A(0)<A\left(x_{i}\right)$ then

Stop:=true;

EndIf

$j:=1$;

While $j \leq|X|$ and not (Stop) do $k:=1$;

While $k \leq|X|$ and not (Stop) do

If $A\left(x_{i} * z_{k}\right)<\min \left(A\left(\left(x_{i} * z_{k}\right) *\left(y_{j} * z_{k}\right)\right), A\left(y_{j}\right)\right)$ then Stop:=true;

EndIf

Endwhile

Endwhile

Endwhile

If Stop then

Output (" $A$ is not a fuzzyP-ideal of $X$ ")

Else

Output (" $A$ is a fuzzyP-ideal of $X$ ")

EndIf

End

Algorithm for Fuzzy $\mathbf{n}$-fold P-Ideals of BCI-Algebra

Input $(X: B C I$-algebra, *: binary operation, $A$ : the fuzzy subset of $X)$;

Output(" $A$ is a fuzzy n-fold P-ideal of $X$ or not");

Begin

Stop:=false,

$i:=1$;

While $i \leq|X|$ and not (Stop) do

If $A(0)<A\left(x_{i}\right)$ then Stop:=true;

EndIf

$j:=1$;

While $j \leq|X|$ and not (Stop) do

$k:=1$;

While $k \leq|X|$ and not (Stop) do

$$
\begin{aligned}
& \text { If } A\left(x_{i} * z_{k}^{n}\right)<\min \left(A\left(\left(x_{i} * z_{k}\right) *\left(y_{j} * z_{k}\right)\right), A\left(y_{j}\right)\right) \\
& \text { Stop: }=\text { true; }
\end{aligned}
$$

EndIf

Endwhile

Endwhile

Endwhile 
If Stop then

Output (" $A$ is not a fuzzy $\mathrm{n}$-fold P-ideal of $X$ ”)

Else

Output (" $A$ is a fuzzy n-fold P-ideal of $X "$ )

EndIf

End

Algorithm for N-Fold weak P-Ideals of BCI-Algebra

Input $(X: B C I$-algebra, $I$ : subset of $X, n \in \mathbb{N})$;

Output(" $I$ is ann-fold weak P-ideal of $X$ or not");

Begin

If $I=\phi$ then

go to (1.);

EndIf

If $0 \notin I$ then

go to (1.);

EndIf

Stop:=false,

$i:=1$;

While $i \leq|X|$ and not (Stop) do

$j:=1$;

While $j \leq|X|$ and not (Stop) do $k:=1$;

While $k \leq|X|$ and not (Stop) do

If $\left(x_{i} * z_{k}\right) *\left(y_{j} * z_{k}^{n}\right) \in I$ and $y_{j} \in I$ then If $x_{i} \notin I$

Stop:=true;

EndIf

EndIf

Endwhile

Endwhile

Endwhile

If Stop then

Output (" $I$ is ann-fold weak P-ideal of $X$ ")

Else

(1.) Output (" $I$ is not ann-fold weak P-ideal of $X$ ") EndIf

End

Algorithm for Fuzzy n-Fold weak P-Ideals of BCI-Algebra

Input ( $X: B C I$-algebra, *: binary operation, $A$ fuzzy subset of $X$ );

Output(" $A$ is a fuzzy n-fold weak P-ideal of $X$ or not");

Begin

Stop:=false,

$i:=1$;

While $i \leq|X|$ and not (Stop) do 


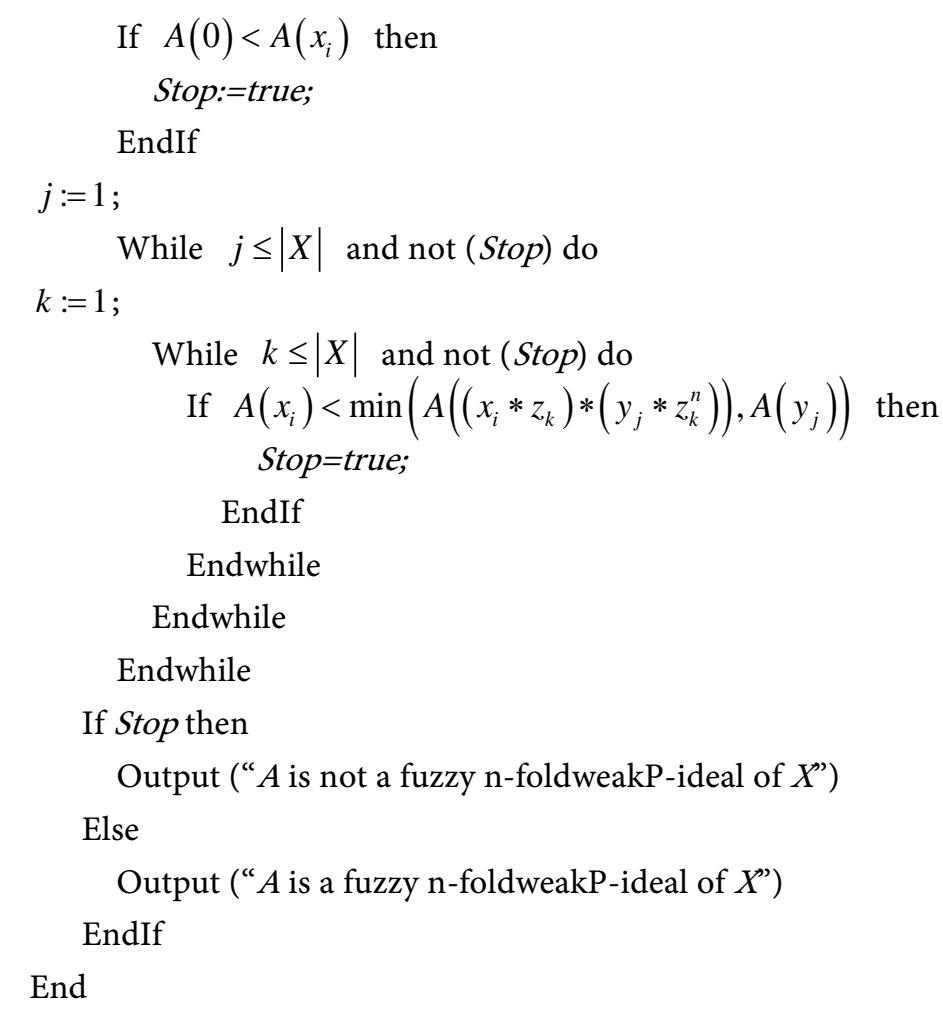

\section{Conclusions and Future Research}

In this paper, we introduce new notions of (fuzzy) n-fold P-ideals, and (fuzzy) $\mathrm{n}$-fold weak P-ideals in BCI-algebras. Then we studied relationships between different type of $\mathrm{n}$-fold $\mathrm{P}$-ideals and investigate several properties of the foldness theory of P-ideals in BCI-algebras. Finally, we construct some algorithms for studying the foldness theory of P-ideals in BCI-algebras.

In our future study of foldness ideals in BCK/BCI algebras, maybe the following topics should be considered:

1) Developing the properties of foldness of implicative ideals of BCK/BCI algebras.

2) Finding useful results on other structures of the foldness theory of ideals of $\mathrm{BCK} / \mathrm{BCI}$ algebras.

3) Constructing the related logical properties of such structures.

4) One may also apply this concept to study some applications in many fields like decision making, knowledge base systems, medical diagnosis, data analysis and graph theory.

\section{Conflicts of Interest}

The author declares no conflicts of interest regarding the publication of this paper.

\section{References}

[1] Iséki, K. and Tanaka, S. (1978) An Introduction to the theory of BCK-Algebra. Ma- 
thematica Japonica, 23, 935-942.

[2] Zadeh, L.A. (1965) Fuzzy Sets. Information and Control, 8, 338-353.

https://doi.org/10.1016/S0019-9958(65)90241-X

[3] Xi, O.G. (1991) Fuzzy BCK-Algebras. Mathematica Japonica, 36, 935-942.

[4] Huang, Y. and Chen, Z. (1999) On Ideals in BCK-Algebra. Mathematica Japonica, 50, 211-226.

[5] Jun, Y.B. (2001) On n-Fold Fuzzy Implicative/Commutative Ideals of BCK-Algebras. IJMMS, 27, 419-427. https://doi.org/10.1155/S0161171201006081

[6] Muhiuddin, G. and Jun, Y.B. (2020) Further Results of Neutrosophic Subalgebras in BCK/BCI-Algebras Based on Neutrosophic Points. TWMS Journal of Applied and Engineering Mathematics, 10, 232-240.

[7] Jun, Y.B. and Meng, J. (1994) Fuzzy P-Ideals in BCI Algebras. Mathematica Japonica, 40, 271-282.

[8] Meng, J., Jun, Y.B. and Kim, H.S. (1997) Fuzzy Implicative Ideals of BCK-Algebras. Fuzzy Sets, and Systems, 89, 243-248. https://doi.org/10.1016/S0165-0114(96)00096-6

[9] Lele, C., Wu, C., Weke, P., Mamadou, T. and Njock, C.E. (2001) Fuzzy Ideals and Weak Ideals in BCK-Algebras. Scientiae Mathematicae Japonicae, 54, 323-336.

[10] Iséki, K. (1975) On Ideals in BCK-Algebras. Mathematics Seminar Notes (Presently, Kobe Journal of Mathematics), 3, 1-12.

[11] Tebu, S.F., Lele, C. and Nganou, J.B. (2012) Fuzzy n-Fold BCI-Positive Implicative Ideals in BCI-Algebras. International Journal of Mathematics and Mathematical Sciences, 2012, Article ID: 628931. https://doi.org/10.1155/2012/628931

[12] Muhiuddin, G., Kim, S.J. and Jun, Y.B. (2019) Implicative N-Ideals of BCK-Algebras Based on Neutrosophic N-Structures. https://doi.org/10.1142/S1793830919500113

[13] Liu, Y. and Meng, J. (2001) Fuzzy Ideals in BCI-Algebras. Fuzzy Sets, and Systems, 123, 227-237. https://doi.org/10.1016/S0165-0114(00)00047-6 